doi: $10.1897 / 05-305 R .1$

\title{
Predicted Effects Of Toxicant Mixtures Are Confirmed By Changes In Fish Species Assemblages In Ohio, Usa, Rivers
}

\section{Leo Posthuma, ${ }^{1, *}$ and Dick de Zwart ${ }^{1}$}

1. National Institute for Public Health and the Environment, Laboratory for Ecological Risk Assessment, P.O. Box 1, NL-3720 BA Bilthoven, The Netherlands

* To whom correspondence may be addressed (E-mail: I.posthuma@rivm.nl)

\begin{abstract}
The purposes of this study were to investigate whether exposure to toxicant mixtures is associated with fish assemblage characteristics in the field and to describe the relationships between predicted chronic and acute mixture risks and observed impacts. Fish abundance and abiotic monitoring data from Ohio, USA, surface waters were compiled and analyzed. Variability of biotic and abiotic parameters was large. Exposure assessment, risk assessment with speciessensitivity distributions, and mixture toxicity rules were used to calculate a relative risk predictor: The multisubstance potentially affected fraction of species (msPAF). Predicted acute and chronic risks ranged from low values to more than 10 and $50 \%$ of species potentially affected, respectively. Pearson correlations between predicted risk and observed assemblage characteristics were nonsignificant for total abundance, number of species, Shannon-Weaver index, and evenness. Moderately significant correlations were found between predicted risk and abundance for $23 \%$ of individual species. Both abundance increases and decreases were observed. Generalized linear model (GLM) regressions revealed significant nonlinear associations between predicted risk and the abundance for $50 \%$ (metals and ammonia) and 55\% (household product ingredients) of the species. Local ecological impact was expressed as the fraction of species expected but not observed, both with and without attribution of impact to mixture exposure. The association between predicted impacted fraction and the fraction of species expected but not observed was not significant. Predicted acute and chronic impacted fractions were associated significantly with the observed fraction of species likely lost by the action of toxicant mixtures under field conditions, with wide confidence bounds. These findings confirm the view that higher mixture impacts are expected in the field at higher msPAF.
\end{abstract}

\section{INTRODUCTION}

A major challenge in risk assessments for toxic compounds is spatially explicit and meaningful quantification of risks to enable focus of risk managers on key problems [1]. The species-sensitivity distribution (SSD) concept can help to address this challenge. The basic assumption of the SSD concept is that laboratory-derived sensitivities of a set of species follow a statistical distribution that can be used to predict the potentially affected fraction (PAF) of species in the field [2]. The available ecotoxicological data determine the moment parameters of the distribution as depicted in Figure 1. 


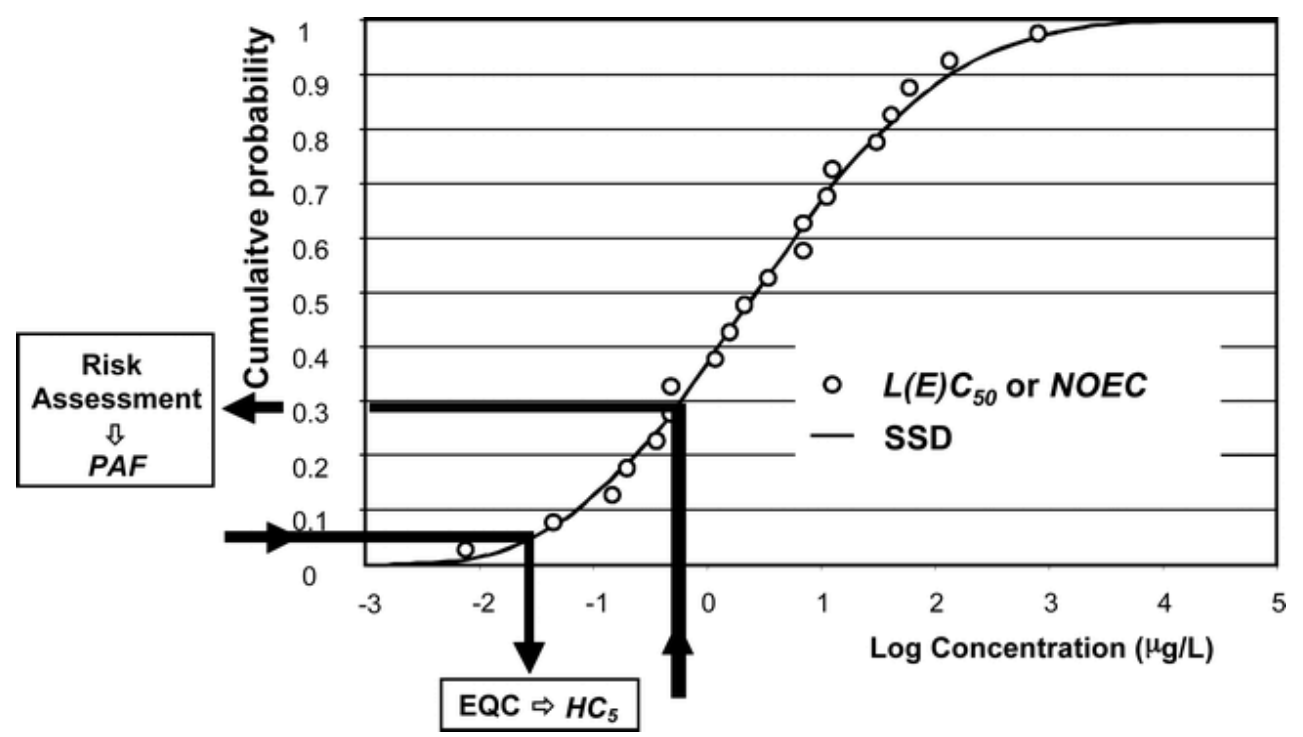

Fig. 1. The species-sensitivity distribution (SSD) concept. (O) Input data (laboratory ecotoxicity data for a compound for a set of species); (-) fitted SSD. The potentially affected fraction of species (PAF) is derived from an ambient concentration $x(x \rightarrow y)$. Environmental quality criteria (EQC) are derived from a chosen value of $y\left(y \rightarrow x\right.$, here $H C_{5}$; e.g., [3]). $\mathrm{L}(\mathrm{E}) \mathrm{C} 50=$ lethal (or effect) concentration yielding $50 \%$ effect; $\mathrm{NOEC}=$ no-observed-effect concentration; $H C_{5}$ $=$ hazardous concentration for $5 \%$ of the species.

Two forms of use of SSDs already were proposed by Van Straalen and Denneman [3]. In practice, SSDs have been used most for the derivation of environmental quality criteria, but more recently use evolved for site-specific quantification of toxic risk in contaminated ecosystems [2]. Klepper et al. [4] introduced the concept of the PAF of species for separate compounds, and Traas et al. [5], Posthuma et al. [6], and De Zwart and Posthuma [7] introduced the multisubstance PAF (msPAF) to quantify predicted mixture risks. Mapping of predicted risks with a geographical information system, and deriving riskmanagement conclusions from such data, could be an answer to the challenge mentioned above. These analyses can be extended to encompass other stressors to acknowledge their possible impacts.

Species-sensitivity distributions currently have large implications in legislation and risk management. Therefore, they are being discussed intensively [2]. Critical issues are fundamental (e.g., the approach is statistical rather than ecological) and technical (e.g., the required number of ecotoxicity data). It is largely unknown to what extent PAF and msPAF can be considered predictors of mixtures' impacts on field communities. This weakness motivates the need for model confirmation studies. Confirmation has been defined as a restricted validation approach, whereby it is investigated whether model predictions and observations are related, and it is acknowledged that the model does not represent the true processes (like in SSDs; see Oreskes et al. [8]).

The SSD confirmation studies [9-16] mainly focused on the fifth centile of SSDs constructed from chronic no-observed-effect concentration values $\left(\mathrm{SSD}_{\mathrm{NOEC}}\right)$, i.e., the hazardous concentration for $5 \%$ of the species $\left(H C_{5}\right)$. Adverse effects on species assemblages usually were observed at (substantially) higher concentrations than the $H C_{5}$ (e.g., $\left.[12,16]\right)$, which matches with the general protection objective of the use of $\mathrm{HC}_{5}$-values in setting quality criteria [2]. The set 
of confirmation studies allows only for a restricted interpretation. Broader confirmation is needed due to the use of SSDs for retrospective risk assessments of contaminated sites. The limitations are as follows. First, the general finding that the predicted $H C_{5, N O E C}$ is lower than the observed NOEC $C_{\text {Ecosystem }}$ is true for any prediction method that systematically is conservative. Second, confirmation studies should test whether whole SSDs match field responses, because exposure often exceeds the $\mathrm{HC}_{5}$ level, and SSDs can be shifted left or right compared to field response curves, are nonlinear, and can have different slopes. Third, various authors $[13,15]$ have shown that several measures of effect can be used to quantify field responses (e.g., total density, total number of species, ShannonWeaver diversity index), so that one SSD prediction curve might be compared to various measure-of-effect curves from field observations. Fourth, SSDs can be constructed from chronic NOEC values, or from acute median effective or lethal concentration (EC50 or EL50, respectively) values, or any other chosen toxicity criterion. Species-sensitivity distributions constructed from these criteria yield an array of possible SSDs, with different ecological interpretations. Fifth, confirmation studies have not yet focused on community effects of mixtures of compounds in multiple stress conditions, i.e., true field conditions.

All this implies that the ecological meaning of an environmental concentration that exceeds the fifth (or any other) centile of an SSD is still unclear, as is the implication of the presence of mixtures. Can the predicted values of PAF $_{\text {NOEC }}$, $\mathrm{PAF}_{\mathrm{EC} 50}$, or msPAF be interpreted in terms of changes in species abundance, species losses, or changed ecological function in the field and, if so, how?

The purposes of this study were to investigate whether the exposure of fish communities in Ohio surface waters to toxicant mixtures is associated with fish assemblage characteristics in the field and, if so, to describe the relationships between predicted chronic and acute mixture risks and observed impacts. Monitoring data on abiotic conditions and fish species abundance in Ohio surface waters were collected and analyzed as described in an eco(logical)epidemiological study [17]. In that paper, emphasis was put on multiple stress analysis, identification of local impacts and their probable causes, and clear georeferenced presentation of results for communicating impact and probable causation to water authorities. The concept of effect and probable cause (EPC) pie diagrams was developed for that paper (Fig. 2A), and involved a multidisciplinary analysis of a large monitoring database (Fig. 2B). The magnitudes of local impacts on fish assemblages and their probable causation were mapped (Fig. 2C). The former paper did not address model confirmation for SSDs, because there was no attention for the relationship between predicted risk and degrees and types of local impacts. The present paper focuses on model confirmation of the SSD model for the prediction of toxic risk in a context of multiple stress. To this end, the characteristics of the monitored fish assemblages were quantified by various parameters. Correlation analyses as well as multiple stress analyses between predicted risks and observed impacts were performed. Special attention was paid to the association between the predicted fraction of species to be affected by toxicant exposure and the fraction of species missing from the local assemblages. 


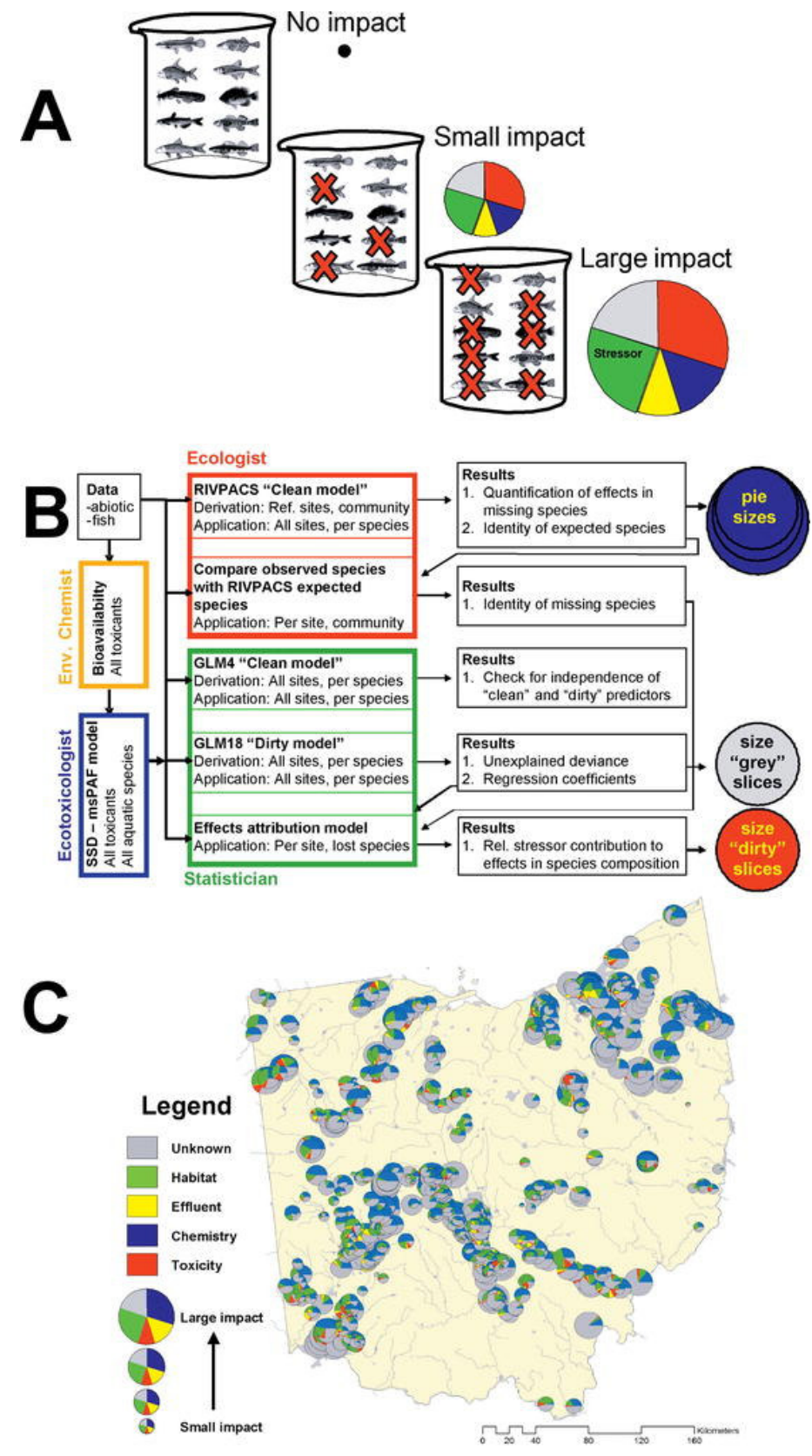

Fig. 2. (A) The concept of effect and probable cause (EPC) diagrams, showing impact per site (pie size, based on fraction of species observed that was expected, $O / E$ ), association of those impacts to the local predictors (slice sizes, different gray tones represent different stressors), and statistical error. (B) Steps in the multidisciplinary data analysis needed to derive EPC diagrams from monitoring data and various models. (C) End result for the Ohio, USA, monitoring data set: Mapped EPC diagrams for 695 sampling locations. Shades represent different groups of stressor parameter types. The graph only serves to illustrate the method, but not to present results. The method defined two impacts expressed as observed affected fraction (with and without assignment of probable causation). 


\section{MATERIALS AND METHODS}

The major components for the current study are the monitoring data set of Ohio rivers (collection, parameters, and handling) the quantification of predicted risk of toxicant mixtures (msPAF) per site, the quantification of observed impacts (with and without assignment of likely causation), and the confirmation studies. The confirmation studies aimed to determine the degree of association between model predictions and field observations.

The earlier study [17] presented details on monitoring, risk quantification, and impact quantification, but not on confirmation. Information from the earlier study is repeated only in short when it is essential for the purpose of describing the confirmation studies.

\section{Monitoring data set of Ohio rivers: Collection, parameters, and handling}

The monitoring data set consists of biological, geographical, physical, and chemical data. Biological data consisted of local abundance data for 117 (98 native and 19 introduced) fish species. Abiotic baseline data for Ohio rivers were compiled [18]. Habitat data included sampling location (latitude, longitude), drainage area above each sample site, vertical gradient (slope), and habitat characterization metrics scored by expert judgment (see [19]). The data included the locations of wastewater treatment plants [20]. Mean flow data were combined with effluent data from wastewater treatment plants to estimate dilution factors and the cumulative percentage of effluents in the river reaches. Ambient water chemistry data collected were: Dissolved oxygen, hardness, $\mathrm{pH}$, and total suspended solids. Median and 90th centile values were determined per site. Toxicant concentrations were either measured [21] (total metal concentrations of $\mathrm{Cd}, \mathrm{Cu}, \mathrm{Pb}, \mathrm{Ni}, \mathrm{Zn}$, and total ammonia), or modeled by river routing. The GISROUT model [22] and the dilution factors of wastewater treatment plants were used to estimate river water concentrations of cumulative effluent and the concentrations of household product constituents (triclosan, linear alkylbenzenesulfonate, alcohol ethoxylates, alcohol ethoxylate sulfates and boron). After toxic risk quantification (msPAF) for the two subgroups (see Quantification of predicted risk of toxicant mixtures (msPAF) section), the data set contained 18 abiotic variables.

Monitoring usually results in many missing data. Omission of rows and columns with missing data for statistical analyses would mean extreme reduction of the set of complete data. Instead of that, gaps in the data set were filled out by imputation [23]. The imputation linked the biological samples with the most representative habitat and chemistry samples [24]. The number of sampling sites with both fish census data and geographical information (latitude, longitude, slope, and drainage area) was 1,552, of which 695 had complete biological, habitat, and chemical data. Of the 1,552 sites, 114 were designated reference sites $[19,25], 60$ of which had complete data.

\section{Quantification of predicted risk of toxicant mixtures (msPAF)}

The quantification of the predicted risk of toxicant mixtures by mSPAF consisted of site-specific exposure assessment and calculation of single-compound PAFs and mixture msPAFs. For heavy metals, the toxicity for fish strongly is associated with the dissolved fraction of the metals that is ionized, which is related to water hardness. Field exposure to metals was calculated from the 90th centile $\mathrm{Cd}, \mathrm{Cu}$, $\mathrm{Ni}, \mathrm{Pb}$, and $\mathrm{Zn}$ concentrations, the median local water hardness and hardness correction factors [26]. The modeled critical low-flow concentrations of household 
product chemicals boron, alcohol ethoxylates, alcohol ethoxylate sulfates, linear alkylbenzenesulfonate, and triclosan were considered as entirely bioavailable. Unionized ammonia is about two orders of magnitude more toxic than the ammonium ion $\left(\mathrm{NH}_{4}^{+}\right)$, and ionization depends on $\mathrm{pH}$ and temperature [27]. Unionized ammonia was calculated from the 90th centile value of measured total ammonia, using site-specific median $\mathrm{pH}$ and assuming a constant temperature of $12^{\circ} \mathrm{C}$.

Species-sensitivity distributions were used to estimate toxic risk (PAF) for each compound. The data for the species-sensitivity distributions consisted of laboratory acute aquatic toxicity test data (EC50), which were obtained from the U.S. Environmental Protection Agency Ecotox database ([28];

www.epa.gov/ecotox/). The SSD EC50 curves could be constructed in this way for ammonia and heavy metals. The $\mathrm{SSD}_{\mathrm{EC} 50}$ curves for surfactants (linear alkylbenzenesulfonate, alcohol ethoxylates, alcohol ethoxylate sulfates) were obtained from a quantitative structure activity relationship-based procedure [29]. Log-logistic functions, in which a specifies the median and $\beta$ the slope [30], were fitted to these data. Chronic NOEC toxicity data were too scarce to construct directly the SSDs. Therefore, chronic SSDs were estimated by applying an assessment factor of 10 (i.e., a left shift of $\mathrm{SSD}_{\mathrm{EC} 50}$ to obtain $\mathrm{SSD}_{\mathrm{NOEC}}$, [30]). The PAF values for individual compounds within both industrial chemicals and household product chemicals were combined to derive separate msPAF $_{\mathrm{NH} 3 \& \text { Metals }}$ and $\mathrm{msPAF}_{\mathrm{HH}}$ values and $\mathrm{msPAF}_{\text {Overall, }}$ both for acute and chronic exposure.

All compounds were assumed to have different toxic modes of action, so that the risk of the mixtures was calculated using the response-addition model. Thereby, it was assumed that species were independent in their sensitivity for the different toxicants [7].

\section{Quantification of observed impacts, with and without assignment of causation}

Observed impacts were quantified with classical biodiversity parameters and as fractions.

Classical biodiversity parameters, no assignment of causation.

Characteristics of local fish communities were quantified with various classical summary parameters, namely species richness index $(S=$ number of species in the sample), total abundance ( $n=$ total number of individuals in the sample), the Shannon-Weaver diversity index [31] $\left(H=-\Sigma\left[p_{i} \cdot \log _{2} p_{i}\right]\right.$, where $p_{i}$ is the proportion of species $i$ in the sample), and the Shannon equitability (evenness) index [31] $\left(E_{H}=H / \log _{2} S\right)$.

Impact quantification based on fractions affected. Impact quantification in terms of impacted fraction of species was done in two ways, with and without assignment of probable causality.

Comparison of the observed fauna with that expected to occur in the absence of anthropogenic stress provided the basis for quantifying local impact. A river invertebrate prediction and classification system (RIVPACS)-type ecological model $[32,33]$ was built from the 114 reference sites data, using latitude, longitude, slope, and drainage area as predictors, to predict probability of capture $\left(P_{\mathrm{c}}\right)$ per species per site. The model was used to estimate the number and identities of species expected $(E)$ at a site under reference conditions. At each sampling site, the observed number of species $(O)$ is known from the sampling. The $O / E$ ratio (calculated on an absolute scale of $0-1$ ) provided the indicator of 
overall impact of all stressors and site conditions, i.e., the fraction of species present that were expected. For the EPC pie diagram presentation of Figure 2C, the magnitudes of impact were calculated as $(1-O / E)$, whereby large radius indicates poor biological condition. This approach is a classical way of RIVPACS modeling, which quantifies impacts, but does not identify the fraction of fish species lost due to specific causes. For this paper, the resulting variable was overall impacted fraction of species per site, $O / E_{\text {all impacts. }}$.

To address probable causation, we developed the method to quantify the fractions of impact on species that likely is attributable to mixture exposure and other potential stressors (slice sizes). The determination of this fraction per site involved ecotoxicological (SSDs) and eco-epidemiological modeling (see Fig. 2AC, details in De Zwart et al. [17]).

To quantify the relationships between different stress parameters and impact, we used generalized linear models ([GLM], [34]). The resulting GLM models quantified the association between the abundance of each of the fish species and the abiotic predictors (GLM18). The modeling converged for 96 species. The GLM18 models were forced to include the four natural variables that were used in the RIVPACS model. Both linear and quadratic forms of the habitat- and contaminant-related variables subsequently were added to the models by a stepwise procedure only when significant $(p<0.05)$. The quadratic forms of the variables were added to enable optimum responses (as expected for, e.g., $\mathrm{pH}$ ). The msPAF NOEC $_{\text {Was }}$ used as a summary variable for the toxicants in the GLMs. Its use improved the statistical power by reduction of the number of parameters in comparison to modeling separate compound concentrations. Significant association between abundance (per species) and a stressor was interpreted as probable cause of impairment. We use the term cause in this restricted sense, although we recognize that statistical associations do not necessarily imply causation. The resulting GLM models summarize the abundance variability across sites in relation to stressor variation, but they do not quantify the impact on local communities.

The RIVPACS modeling results were used to provide the identities of species that were expected but missing per site. By looking at the subsets of missing species per site, it became possible to generate the EPC pie diagrams (Fig. 2A and Fig. 2C) as follows. If a species was concluded missing at a particular site, the separate contributions of the different stressor variables in the GLM18 model prediction were considered. A contribution is defined as the net value of the sum of the linear and the quadratic terms for a parameter. The GLM18 regression models were used to predict the numerical abundance of species $i$ at a site $\left(E_{i, G L M 18}\right)$ by filling out the local values of both the naturally occurring and stressor variables in the formulae. For a missing species at a site, one or more contribution terms must be negative, otherwise GLM18 would not be able to predict the local species' absence. If species $i$ was missing at site $x$ and the GLM18 model predicted a negative influence on the abundance of that species at the site for a particular parameter (e.g., msPAF), this was considered evidence that the parameter (e.g., the mixture of chemicals) contributed to the species' absence. Consideration of all the parameters, and all cases with a negative contribution of a parameter to the local abundance of a species according to its GLM18, yielded site data sets of negative contributions. Using this data set, the relative potential influence of each stressor variable per site was calculated as that stressor's negative contribution divided by the sum of all negative stressor contributions for the species. These relative proportions, along with unexplained variance, were used to size the pie slices in the EPC diagrams. Unexplained variance in species abundances at each of the sites was calculated as the 
departure from a linear association between observed and expected ( $\left.E_{i, G L M 18}\right)$ abundances over all species. Unexplained variance, including unknown causes and model error, was quantified as $1-r^{2}$ and included in the EPC diagrams as one of the slices. The information for the different variables along with the unexplained variance was used to size the pie slices in the EPC diagrams.

For the confirmation study, the final step was to calculate the fraction of species probably lost due to the toxic action of the considered chemicals. This fraction was calculated from the magnitude of overall impact $(O / E$, local pie size, obtained from RIVPACS, representing the fraction of lost species) multiplied by the relative contribution of toxic risk (slice size associated with MSPAF, as defined by combined RIVPACS and GLM modeling). For this paper, the resulting variable was toxicant-impacted fraction of species per site, $O / E_{\text {toxicity impacts. }}$

\section{Confirmation: Associations between model predictions and field observations}

The associations between model predictions (msPAF values for the sites) and various parameters for quantification of field responses were determined. The analyses that were performed represent an array of methods with increasing specificity in the way they summarize the observed assemblage characteristics. This was done because it was expected from the previous analyses that simple association approaches for highly aggregated impact parameters (like the Shannon-Weaver index) likely would yield nonsignificant relationships due to the dominant role of other stressors. The Ohio summary presentation of an effect and probable cause diagram [17] showed that the combined toxicity of metals, ammonia, and household product chemicals were associated with on average $3 \%$ of the biological degradation. The confirmation studies consisted of Pearson product-moment correlation analyses, multiple stressor analyses (GLM), and regression analyses of the impacted fraction of species to the predicted value of the model (msPAF), with and without assignment of probable cause.

\section{RESULTS AND DISCUSSION}

\section{Variation in predicted toxic risk (msPAF) and other parameters across Ohio}

Substantial variation was present in the data set of abiotic variables (Table 1 ). Correlations among variables were determined, because the interpretation of the confirmation studies might be hampered by such correlations. Correlations between parameters were reported in detail in De Zwart et al. [17]. The correlations between mSPAF and other abiotic parameters appeared to be nonsignificant.

Despite the low average contribution of toxic risks of mixtures (3\%) to the overall impacts on fish assemblages in Ohio [17], there is substantial variation in predicted risks (msPAF) among the sampling sites. A steady increase of mixture risks across sites was observed, with slightly lower representation of higher values (Fig. $3 \mathrm{O}=$ ). In approximately $10 \%$ of the sites (proportion of sites $>0.9$ ), the mixture exposure is predicted to induce minor chronic effects (NOEC exceedance) in $50 \%$ of the species, and it is predicted that the acute median effect level (EC50) at these sites is exceeded for approximately $10 \%$ of the species. Thus, the prediction methods suggest that an array of local effects is to be expected, ranging from negligible chronic to substantial acute effects. 


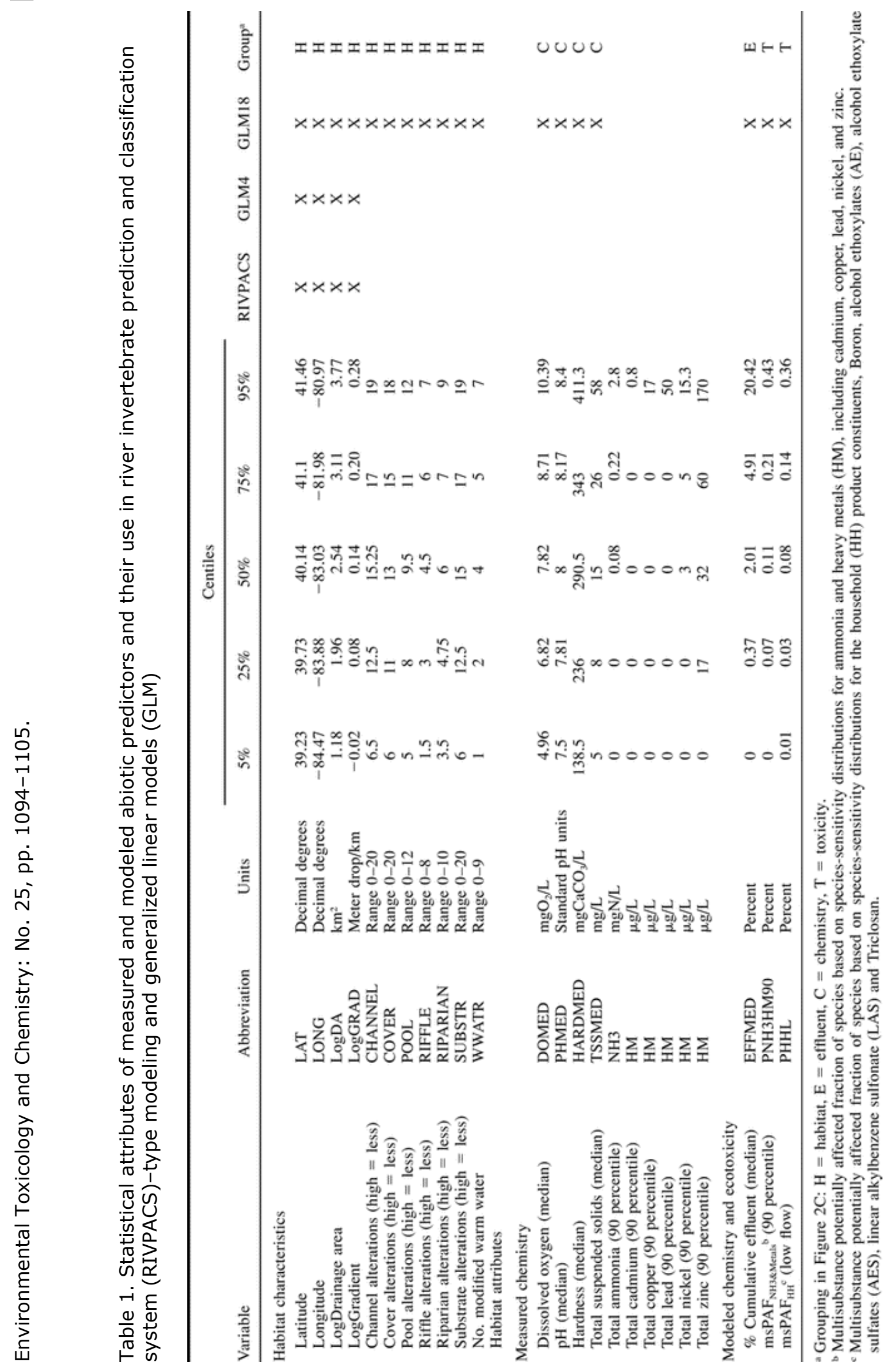




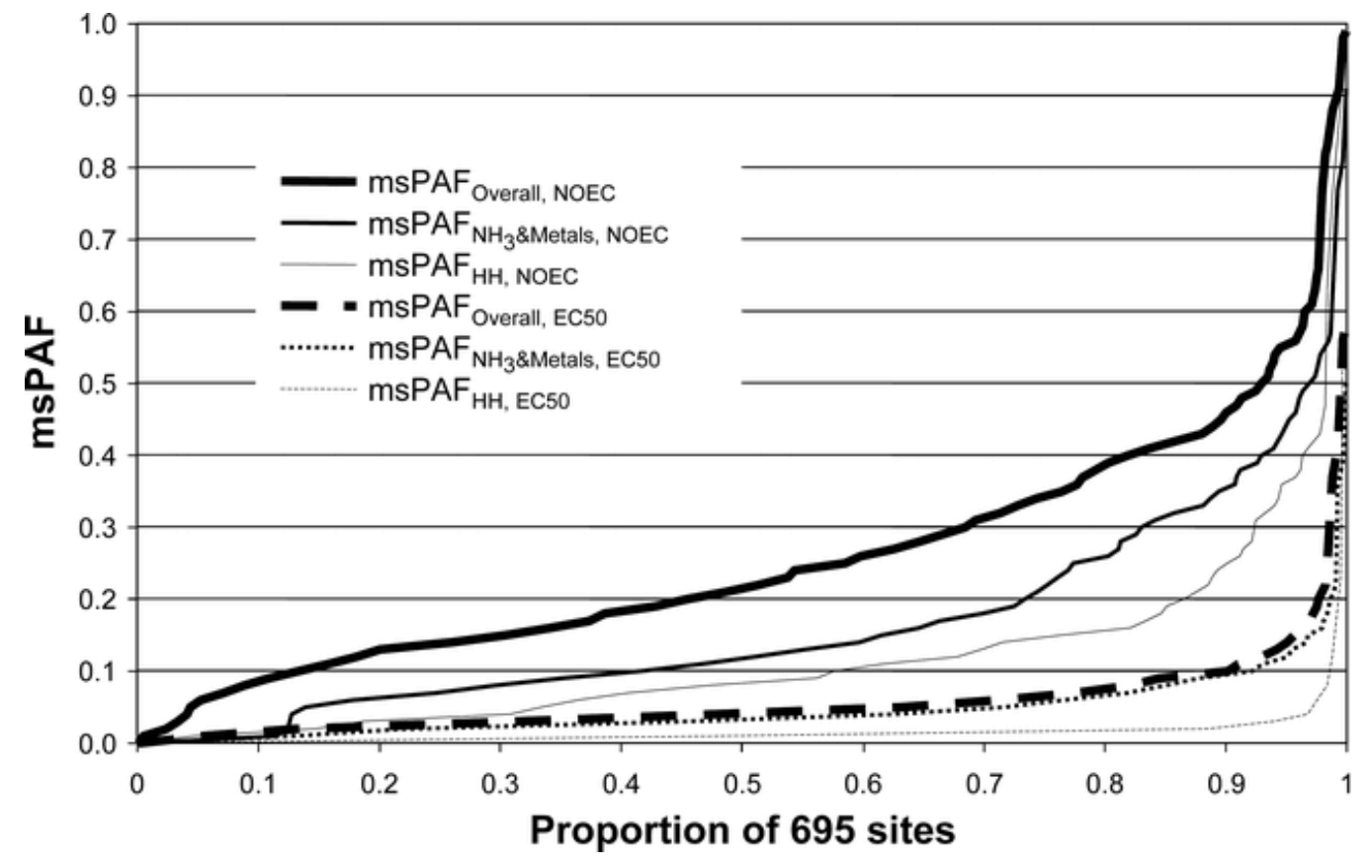

Fig. 3. Cumulative profile plot for sites versus chronic and acute msPAF. For $\mathrm{PAF}, \mathrm{NOEC}, \mathrm{EC50}$, see Figure 1. $\mathrm{mSPAF}_{\mathrm{NH} 3 \mathrm{~s} \text { Metals }}=$ multisubstance PAF associated with concentrations of ammonium and metals; msPAF $_{\mathrm{HH}}=$ multisubstance PAF associated with the concentrations of household products; the subscript NOEC or EC50 refers to sets of ectotoxicity data used for constructing $S_{S D_{\text {NOEC }}}$ or SSD $_{\text {EC50 }}$.

The relationship between acute and chronic mixture risks is strong ( MSPAF $_{\mathrm{EC} 50}$ $=0.24 \cdot \mathrm{msPAF}_{\mathrm{NOEC}} ; r^{2}=0.66, p<0.001$ ), but it is not a uniform factorial difference. This is a result of the nonlinear characteristics of SSDs. Therefore, both types of msPAF are considered separately in the confirmation studies.

De Zwart et al. [17] reported substantial variation for all abiotic parameters. Fifty percent of the biological effects was associated with variation in the measured abiotic parameters, most of which was related to alterations in the parameters for general water chemistry and habitat characteristics ( 28 and $16 \%$, respectively). This implies that these factors, on average, are dominant over mixture toxic risks (on average: $3 \%$ ) in shaping fish community structures. In turn, this implies that refined methods (site- and stressor-specific) may be needed in the confirmation studies.

\section{Variation in the occurrence of species across Ohio}

De Zwart et al. [17] reported that the RIVPACS model accounted for $51 \%$ of the variation in observed natural variation in species composition and richness among reference sites. This means that latitude, longitude, drainage area, and slope are major determinants of species occurrence. In turn, this implies that the species sets that are expected to occur differ among sites. Therefore, the confirmation studies need to take into account that impacts are not expressed on the basis of a standard set of expected species, but relate to site-specific assemblages of species with likely different tolerances and sensitivities. It is known that tolerances and sensitivities differ between species $[35,36]$. 
Environmental Toxicology and Chemistry: No. 25, pp. 1094-1105.

Table 2. Correlations between predicted chronic risk ( $\mathrm{mSPAF}_{\text {Overall,NOEC }}$ ) and observed fish assemblage characteristics as quantified by classical biodiversity parameters. Number of observations: 695

\begin{tabular}{ll}
\hline Ecological endpoint & Correlation statistics \\
\hline S (total no. of species) & $r^{2}=3.010^{-6}(p>0.05)$ \\
N (total no. of individuals) & $r^{2}=2.010^{-4}(p>0.05)$ \\
H (Shannon-Weaver index) & $r^{2}=1.510^{-3}(p>0.05)$ \\
E (Shannon's equitability index) & $r^{2}=5.010^{-3}(p>0.05)$ \\
\hline
\end{tabular}

\section{Confirmation}

Pearson correlations. Associations between predicted chronic risk ( $\mathrm{MSPAF}_{\text {Overall,NOEC}}$ ) and classical assemblage-level biodiversity metrics all were nonsignificant (Table 2). Pearson product-moment correlations between predicted chronic risk and observed species abundance could be made for 97 species and were significant $(p<0.05)$ for $23 \%$ (i.e., 25 of the species;Fig. 4$)$. For this figure, the correlation coefficients between predicted chronic risks and the abundances per species were calculated for all species and thereafter ranked. For both groups of toxicants combined, there are approximately as many species $(10 \%, 11$ species) increasing in abundance with increasing toxicity (apparently opportunist species) as there are species (13\%, 14 species) decreasing in abundance (apparently sensitive species). Abundance increases in part might be attributed to hormetic effects (stimulating effect of low exposure), but likely represent indirect effects, where removal of sensitive species provides a niche for tolerant opportunist species. The lowest and highest correlation coefficients were -0.14 and +0.15 , respectively. The correlation coefficients were nonsignificant ( $p$ $>0.05$ ) for the remaining species.

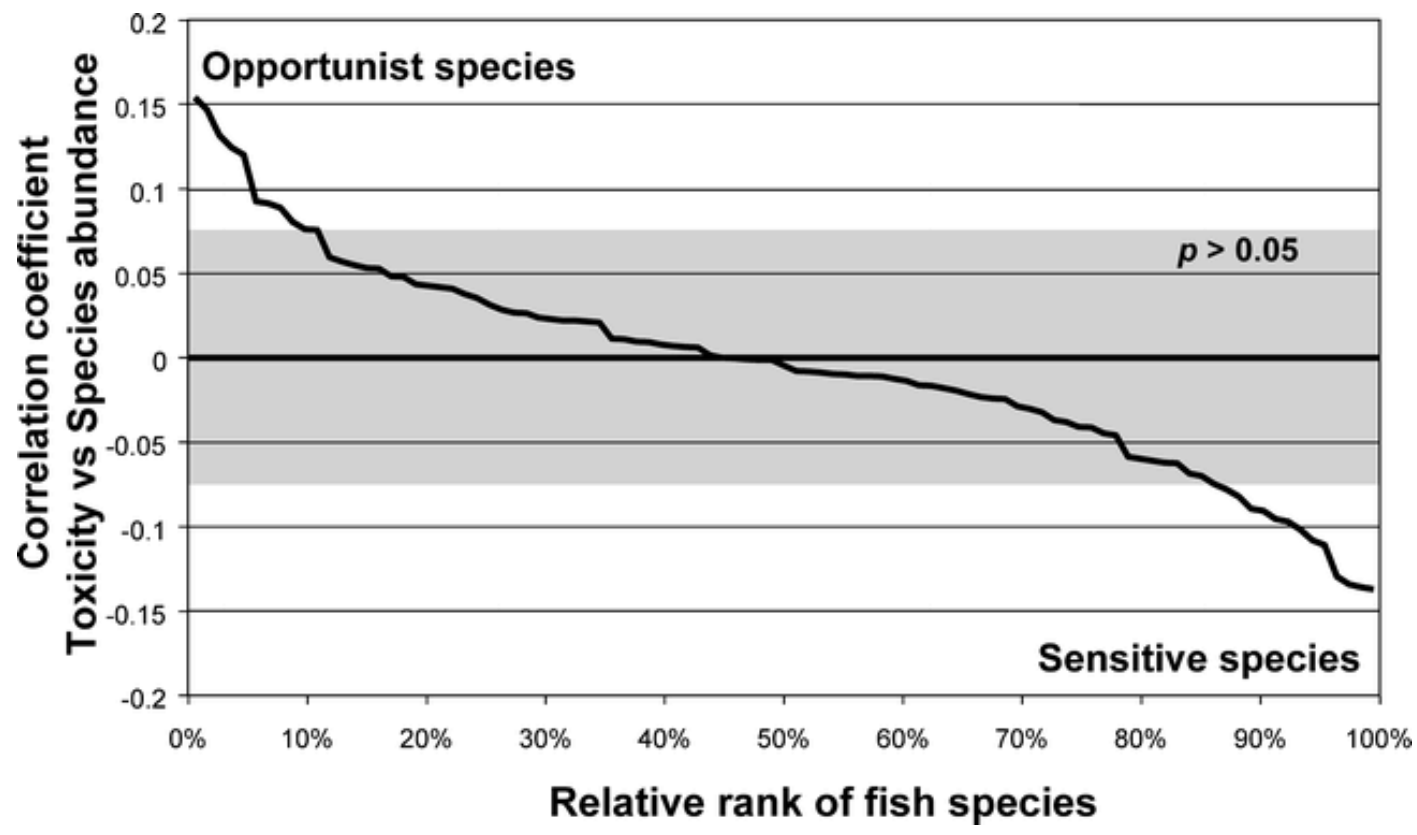

Fig. 4. Species-ranked presentation of the 96 product-moment correlations between chronic risk ( MSPAF $_{\text {Overall,NOEC }}$ ) and the abundance of individual fish species. Gray area indicates nonsignificant correlations $(n=695, p>0.05)$. 
The correlation analyses demonstrated that there is association between predicted risks and observed impacts only when looking at species-level response patterns, given the range of mixture exposure in the data set. Assemblage-level impact parameters for this data set did not relate to predicted impact, as a probable consequence of both increased and decreased abundances with increased mixture exposure. For confirmation, these results imply that increases in predicted risks are associated with changes in the abundance of a moderate number of species $(-23 \%)$ over the whole data set. This is a relevant conclusion regarding presence of impact, but it only partially confirms the mSPAF as predictor of impact: These analyses do not allow for quantification of impacts in the format of a mixture-affected fraction of species per site.

Table 3. Summary of the degrees of significance in the generalized linear model with 18 parameters (GLM18) that describe how the abundance data for 96 fish species are associated to the geographical and stress factors in the data set. RSS = residual sum of squares; the subscript NOEC added to the MSPAF variables indicates the SSDs were based on NOECs. See Table 1 for additional variable abbreviations

\begin{tabular}{|c|c|c|c|c|c|c|c|}
\hline \multirow[b]{2}{*}{ Predictor variables } & \multirow{2}{*}{$\begin{array}{l}\text { Predictor } \\
\text { occurs in } \\
\text { percentage } \\
\text { of species }\end{array}$} & \multicolumn{6}{|c|}{$\begin{array}{l}\text { Significance of regression terms related to increase of RSS upon re- } \\
\text { moval from the final GLM18 models per species }(\%)\end{array}$} \\
\hline & & $p \leq 0.001$ & $\begin{array}{l}0.001<p \\
\leq 0.01\end{array}$ & $\begin{array}{l}0.01<p \\
\leq 0.05\end{array}$ & $\begin{array}{l}0.05<p \\
\leq 0.1\end{array}$ & $\begin{array}{l}0.1<p \\
\leq 0.5\end{array}$ & $\begin{array}{l}0.5<p \\
\leq 1.0\end{array}$ \\
\hline LAT & 99 & 71 & 7 & 3 & 2 & 11 & 6 \\
\hline LONG & 96 & 64 & 7 & 3 & 3 & 23 & 0 \\
\hline LogGRAD & 94 & 52 & 7 & 9 & 6 & 27 & 0 \\
\hline LogDA & 98 & 72 & 3 & 4 & 2 & 18 & 0 \\
\hline DOMED & 45 & 86 & 5 & 5 & 0 & 5 & 0 \\
\hline HARDMED & 55 & 85 & 13 & 0 & 0 & 2 & 0 \\
\hline EFFMED & 65 & 92 & 6 & 0 & 0 & 2 & 0 \\
\hline PHMED & 43 & 95 & 5 & 0 & 0 & 0 & 0 \\
\hline TSSMED & 67 & 89 & 11 & 0 & 0 & 0 & 0 \\
\hline CHANNEL & 47 & 96 & 2 & 0 & 0 & 2 & 0 \\
\hline COVER & 66 & 92 & 5 & 3 & 0 & 0 & 0 \\
\hline WWATR & 48 & 91 & 9 & 0 & 0 & 0 & 0 \\
\hline POOL & 46 & 86 & 14 & 0 & 0 & 0 & 0 \\
\hline RIFFLE & 47 & 87 & 9 & 2 & 0 & 2 & 0 \\
\hline RIPARIAN & 44 & 88 & 12 & 0 & 0 & 0 & 0 \\
\hline SUBSTR & 50 & 88 & 8 & 4 & 0 & 0 & 0 \\
\hline $\mathrm{mSPAF}_{\text {HH,NOEC }}$ & 55 & 91 & 8 & 0 & 2 & 0 & 0 \\
\hline $\mathrm{msPAF}_{\mathrm{NH} 3 \& \text { Metals,NOEC }}$ & 50 & 92 & 6 & 0 & 2 & 0 & 0 \\
\hline
\end{tabular}

Multiple stressor GLM analyses. When the abundances of separate species apparently relate in part to the predicted mixture impact, this must show up in the GLM18 formulae. The use of these formulae allows for multiple stressor analyses and acknowledges that nonlinear association may be present in the form of optima, and with Pearson correlations it is assumed implicitly that there are only linear associations. Detailed analysis of the GLM18 formulae showed that there are highly significant associations between abundance variation of individual fish species over Ohio and the geographical or stressor variables (Table 3 ). The parameters with an associated linear response in most of the species ( $>90 \%$ of the species) are latitude, longitude, drainage area, and gradient, but these variables were forced linearly into the GLM18 calculations. For the other variables, which either could be adopted in the GLM18 or not, depending on significance of 
term additions, possibly nonlinear (quadratic terms) responses were found for 43 to $67 \%$ of species. The proportion of species for which chronic risk (msPAF NOEC $_{\text {) }}$ significantly $(p<0.001)$ was associated with their local numerical abundance was $55 \%$ for the low flow concentrations of household product chemicals and $50 \%$ for the 90 th centile metal and ammonia concentrations. The variation in local concentrations of toxicants apparently influenced population densities in a moderate number of species, as have other variables. For confirmation, these results imply that increases in predicted chronic risks are associated with changes in the abundance of half of the species $(-50-55 \%)$ over the whole data set. Again, this only partially confirms the msPAF as predictor of impact because these analyses do not allow for quantification of impacts per site as the fraction of species affected by the combined action of toxicant mixtures.

\section{Impacted fractions of species, with and without attribution to causes}

The predicted risk of toxicant mixtures (MSPAF) is expressed as impacted fraction of species. Therefore, further confirmation studies focused on the observed fractions of species lost from local species assemblages. In these studies, a regression approach was followed $O / E_{i}=a_{i} \cdot \operatorname{msPAF}+b_{i}+\boldsymbol{e}$ where $O / E$ is the affected fraction of species (magnitude of impact), $i$ is the endpoint chosen (with or without causal attribution), $a$ and $b$ are constants for the respective field effect parameter $i$, and $\varepsilon$ is the error. Such functions can be significant or not significant. The prediction method (msPAF) would be confirmed by an associated field impact $\left(O / E_{i}\right)$, that is: When the analyses would show a positive, significant regression between predicted mixture risks and impacts attributable to mixture exposure.

First, the observed impact was quantified directly by the overall $O / E$ scores from RIVPACS. The O/E-score was not associated significantly with msPAF (Fig. $5 A$ and $5 B$ ). It is likely that the lack of significance again has resulted from the complex set of increasing and decreasing abundance responses and the major influences of the other stressors. Note that, when using the overall $O / E$-scores, predictions and observations are completely independent: Laboratory toxicity data, exposure modeling, and the SSD model are used for the prediction of the causal parameter on the $x$-axis (msPAF), and local species counts are used for quantifying the observed local impacts on the $y$-axis (O/E obtained by RIVPACS).

Second, the impact was quantified similarly, but now looking only at the fraction species likely lost due to toxicant mixture exposure $\left(O / E_{\text {toxicity impact }}\right)$, in line with the GLM conclusion that separate attention is needed for msPAF as stressor variable. To this end, the overall $O / E$ score (=pie size) was multiplied by the fraction of impact attributed to mixture toxicant exposure (i.e., slice size for toxicity). This approach yielded significant regressions between msPAF (predictor) and the loss of species attributable to toxicant mixtures for predicted chronic and acute risks (Fig. 5C and 5D). The confidence intervals of both regressions are wide. This reflects the major influence of other stress factors in shaping local fish assemblages, as was apparent already from the GLM18 analyses and from the differences among fish communities under reference conditions (RIVPACS). The regressions suggest that the model predictions (predicted fraction of species affected by mixture exposure) are confirmed by observed field impacts (observed fraction of species affected attributed to mixture exposure). 
Environmental Toxicology and Chemistry: No. 25, pp. 1094-1105.
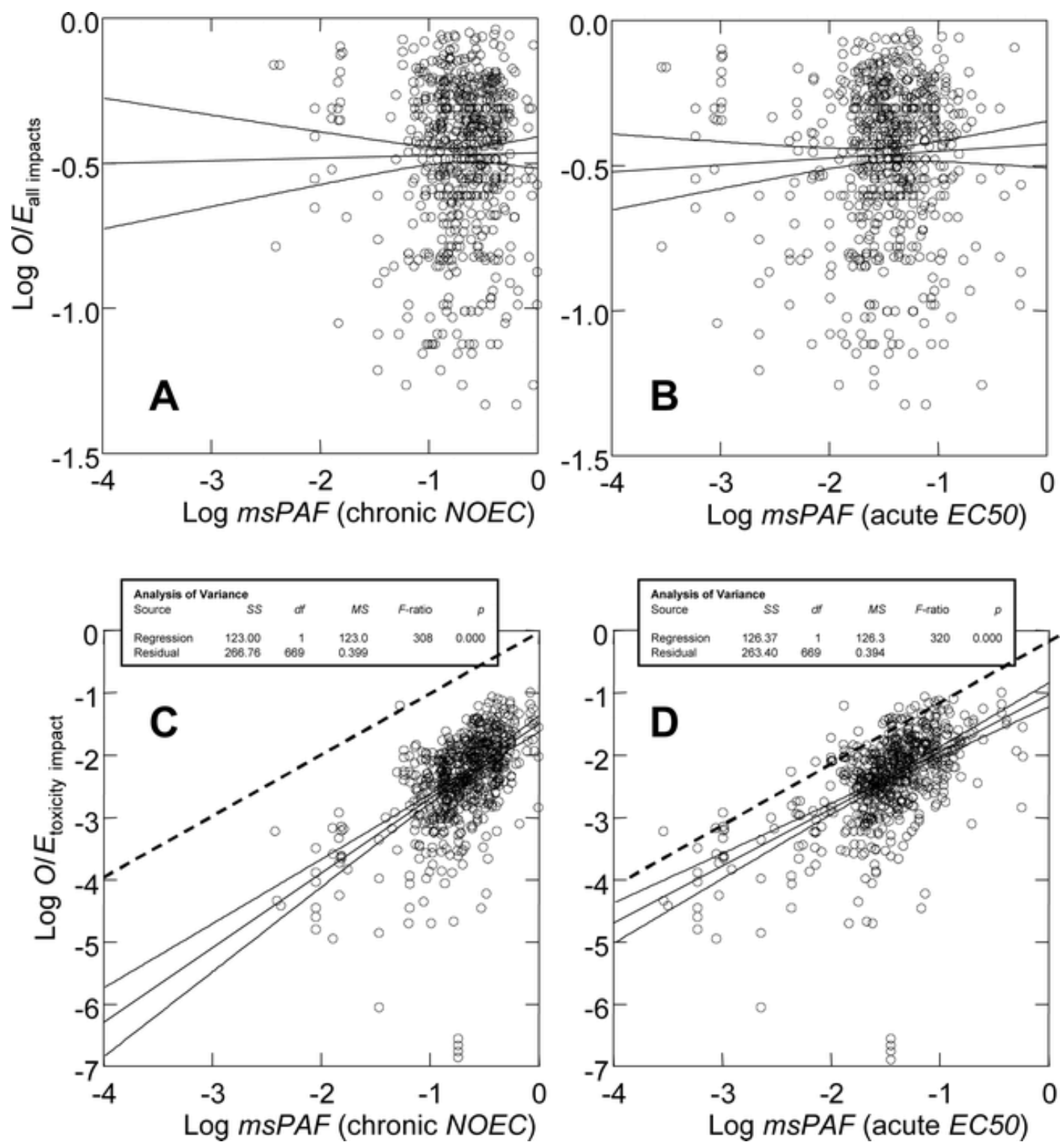

Fig. 5. The regression of observed impact, expressed as observed toxicantaffected fraction of species, on the predicted mixture impact (msPAF). Graphs (A)

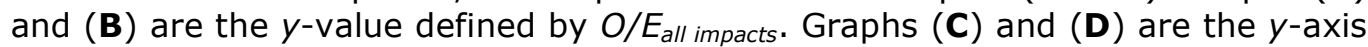
defined by $O / E_{\text {toxicity impact. }}$ Graphs $(\mathbf{A})$ and $(\mathbf{C})$ are the $x$-axis $=$ predicted chronic risk. Graphs (B) and (D): $x$-axis $=$ predicted acute risk. All axes are on a log10 scale. Significant regression statistics presented (for (C) and (D)). The dotted lines indicate the hypothetically ideal 1:1 confirmation relationship, where the observed fraction affected equals the prediction (mSPAF). For NOEC and EC50 definitions, see Figure 1 ; for $\mathrm{mSPAF}$, see Figure 3. O/E = ratio of observed species at a site over the expected species for that site, a quantity obtained from ecological modeling, without $\left(O / E_{\text {all impacts }}\right)$ and with assignment to probable causes $\left(O / E_{\text {toxicity impact }}\right)$.

For the later two regressions, it is noted that there may be regression bias, because the predicted risk (msPAF) plays a role in defining the $x$-axis (predictor, in full) and the $y$-axis (impact variable, in part). Partial circularity of reasoning was unavoidable in the eco-epidemiological analyses; however, inspection of the analysis methods shows that the circularity is not full here. That is, the $y$-value 
calculation involves three elements of independence between $x$ and $y$ parameters. First and second, the magnitudes of local impact $(O / E)$ and the identities of species missing were determined from the field data set through RIVPACS. The RIVPACS modeling solely depended on field data on fish occurrence, not on predicted msPAF. Third, the mixture-related slice size not only is determined by the local value of msPAF, but also by the relative roles of the other independent stressors. This implies a partial and likely nonlinear influence of msPAF on the impact fraction attributed to mixture exposure. We concluded earlier (correlation analyses and GLM analyses) that there are (highly) significant mixture exposure influences on Ohio fish species, and this strongly suggests that the fraction of species affected by mixtures across sites (the $y$-values in Fig. 5C and 5D), on average, also should show a signal. Because of this, we have derived the regressions of Figures $5 \mathrm{C}$ and $5 \mathrm{D}$ to describe how the expected fraction affected relates to the observed toxicant-affected fraction, be it with the notion that the regressions can be influenced by partial (not full) circularity of reasoning.

When it is assumed that Figures $5 C$ and $D$ provide a summary overview of the relationship between predicted and observed mixture impacts, there are some remarkable issues. The slopes of both regression lines are near unity, which is remarkable because calculation of the species loss attributable to toxicant exposure also may contain a nonlinear (quadratic) term for msPAF. Scatter around the regression lines represents the large variability in the data set that was present for all abiotic parameters and for the different distributions of fish species under reference conditions. The regression of observed impacts on acute risks revealed the closest and most uniform match with the hypothetical one-toone relationship (dotted line) expected for a perfect prediction model. This may be due to the more direct way of calculating $\mathrm{SSD}_{\mathrm{EC} 50}$ from the original toxicity data and due to the fact that EC50 exceedance more likely is associated with observable effects than NOEC exceedance. For the $\operatorname{msPAF}_{\mathrm{EC} 50}$ values, nearly all scatter plot data were located below the dotted line. This pattern is likely because it was shown that impact is exerted by multiple stressors. Toxic risk may predict an effect in a particular proportion of species; however, these species already may be affected by any combination of the other stress factors so that their loss is not attributed to toxic stress in the impact attribution calculations. Reasoning suggests that additional loss of species may be induced at higher exposures due to ecological interactions, like the loss of an insensitive predator due to the loss of a sensitive prey species. From the fact that graph $D$ only displays a few instances where data are located above the dotted line, it may be concluded tentatively that such indirect toxic effects do not result frequently in an additional loss of species by indirect effects to a degree higher than the value of the estimated $\mathrm{msPAF}_{\mathrm{EC} 50}$ in the data set. Although the regression in graph $D$ suggests that loss of species attributable to acute toxicity is a factor of 10 lower than predicted acute toxic risk, the scatter plot data reveal that the maximum toxicity-attributed fractional loss of species comes close to predicted acute toxic risk $\left(O / E_{\text {all impacts }}=\right.$ $\left.\mathrm{msPAF}_{\mathrm{EC} 50}\right)$. Ecological considerations suggest that, at low exposure, direct effects might be limited because of ecological compensation mechanisms, whereas at high exposure direct effects can be magnified by changes in ecological interactions [37], e.g., an insensitive predator can suffer from effects on a more sensitive prey [38]. This implies that the relationships between observed and predicted impacts necessarily need not be linear. The results of our analyses, however, insufficiently are clear as to break the linear null model in various ranges (neutralizing, linear, magnifying).

It is possible that scatter around the regression lines can be diminished with an improved prediction model and more complete coverage of possible stress factors (e.g., pesticides). However, natural variation unlikely will be explained completely by modeling. Regarding risk modeling, it is noteworthy, however, that a single 
field concentration conceptually implies the presence of a set of risk predictions, namely the risk of exceedance the EC50 for $5 \%$ of the species also does imply that there also is risk of exceedance of the EC40, EC30, EC20, EC10, and NOEC, for increasing proportions of species. To give a set of risk predictions for one ambient concentration, it is necessary to construct SSDs for various response levels (NOEC, EC10, EC20, etc.). The generation of such a distribution of msPAF values has been considered by Posthuma et al. [6] and was exemplified by Giddings et al. [39] for two data-rich compounds. The resulting distribution of msPAF levels per site in such cases might be associated with the species-specific sets of responses, as depicted in Figure 3 (the increasing and decreasing abundance effects).

External validation of species responses. All results obtained so far are statistical and model results. Validation of these results with other (autecological) species data is possible when considering the species' general stress sensitivity characterizations from literature $[35,36]$. These are based on the frequencies of species occurrences in stressed ecosystems (erosion, channelization, proximity to discharges, etc.). Based on the Pearson correlations, the following species showed an opportunistic response in relation to mixture risks: Stonecat madtom, river chub, brindled madtom, bigeye chub, fantail darter, emerald shiner, freshwater drum, spotted bass, central stoneroller, bluntnose minnow, and green sunfish. Oppositely, the following species can be considered sensitive to mixture risks: Black redhorse, tonguetied minnow, northern hog sucker, mottled sculpin, silver shiner, walleye, black crappie, rainbow darter, northern pike, rock bass, white crappie, bowfin, blacknose dace, and white sucker. In literature, the species were assigned to the general stress classes tolerant ( $T$ ), intermediate (M), and intolerant (I). Some species were assigned to two classes (e.g., M and T), and one species (Silver shiner) had a tolerance classification of I and $\mathrm{T}$. Comparisons of both classifications shows that the classifications were identical for two out of the 25 species, none or one class apart for 14 species, one or two classes apart for six species, two classes apart for two species, and none or two classes apart (the silver shiner). Thus, 16 to 22 classifications were similar or, at most, one class different. Apparently, the assignment of mixture sensitivity by the correlation analyses grossly is associated with the overall assignments of the species' general stress sensitivities.

The analyses of the monitoring data set suggest that SSD-based risk predictions grossly but not perfectly predict ecological field effects. The latter would have been highly surprising due to the lack of ecological considerations in the model and lack of attention for variation in the field. Given the above results and according to the principles of parsimony, however, SSDs may yield useful output for various policy targets. Limitations that follow from the fact that method is a modeling approach, based on statistics (as first principle) and laboratory toxicity data (as operands), with lack of attention for variability and ecological interactions in the field, always should be acknowledged. For the derivation of environmental quality criteria, the requisites of method accuracy, practicality, and relevance apparently have been fulfilled, as substantiated by use in various legislations and supported by various observations that the protection criterion $\left(H C_{5}\right)$ often is below the response threshold (NOEC $\left.\mathrm{Ecosvstem}\right)$ [16]. Currently, the method is used increasingly in the context of the ranking of compounds, sites, and impacts, for example in life-cycle assessments [2]. In view of the latter, further studies of the relationship between predicted impact and observed impacts in the field are valuable to avoid mis- or overinterpretation of predictions. 
Environmental Toxicology and Chemistry: No. 25, pp. 1094-1105.

\section{CONCLUSION}

The results presented in this paper show that exposure to toxicant mixtures in field conditions is associated with altered fish assemblage characteristics, especially for specific parameters derived from species-level abundance and occurrence information. Pattern analyses of the assemblage characteristics have shown that the predicted risk (msPAF) relates to the latter subset of specific impact parameters, although there was large scatter around the regression line between predicted risk and impact assigned to mixture toxicity. The analyses revealed the importance of considering toxicant mixture effects in a multiple stress context. Lack of association between predicted risk and ecological summary parameters likely is due to the highly dynamic set of multiple stressresponse types. Earlier studies $[15,40]$ reported similar differences between effect quantification parameters. In terms of confirmation, this study shows that the statistical and intuitive meaning of msPAF, to a certain extent, is correct: An increase of msPAF is associated with various species-specific effects increase, in a highly significant way and for a variety of species. The observed loss of species that can be ascribed to mixture toxicity closely matches the predicted acute risks $\left(\operatorname{mSPAF}_{\mathrm{EC} 50}\right)$, at least in a relative sense (slope $\left.1: 1\right)$, and with a maximum observed fraction of lost species equal to the predictor variable $\left(\operatorname{msPAF}_{\mathrm{EC} 50}\right)$. For the chronic risk predictor, a similar observation holds, whereby the maximum observed fraction of lost species is approximately one-tenth of the predictor value. Due to these relationships, msPAF as predictor parameter may have the diagnostic properties required to assess ecological condition and to identify toxicity-impaired ecosystems [1]. The degree of response to toxicants thereby highly depends on the responses of the fish species assemblage to other stressors. If a system already has collapsed due to adverse conditions in any of the other stress factors, the contribution of toxicant stress to shaping the local community might be zero. A key, indirect observation of this study is that the use of the msPAF approach can be important to the interpretation of (future) monitoring data. Various jurisdictions (e.g., European Union, United States) implement large-scale monitoring efforts, whereby both impact and causal analyses are requested. Usually, addition of variables (separate compounds) trades off in reduced statistical and inferential power. Using msPAF might help to improve statistical power in data analyses and to find impacts and probable causation. Eventually, use of the msPAF approach may play a role in linking compound regulations (e.g., the European Union's policy on chemical regulation, Registration, Evaluation, and Authorization of Chemicals [REACH]) and regulations targeting at the biological integrity of environmental compartments for which monitoring plays a role (e.g., European Union-Water Framework Directive). This warrants further developments to improve on SSDs both in concept and practice.

\section{Acknowledgments}

This work was funded by the Strategic Research Program of the National Institute for Public Health and the Environment (RIVM), a program under auspices of the RIVM Director and the Scientific Advisory Board, within RIVM (project S860703), and cosponsored by the Dutch Stimulation Program System-Oriented Ecotoxicological Research (project SSEO 014.23.094). Thanks are due to S. Dyer, who made available and preprocessed the raw monitoring data as reported in various earlier works, thus making this work possible and to many coworkers for their efforts in the fieldwork and subsequent sample analyses. We wish to thank C. Mulder, D. van de Meent, T. Aldenberg, S. Dyer, G. Carr, and C. Hawkins for stimulating discussions. The manuscript improved due to the comments of $\mathrm{J}$. Struijs, C. Mulder, and anonymous reviewers. 


\section{References}

1. Bradbury SP, Feijtel TCJ, Van Leeuwen CJ. 2004. Meeting the scientific needs of ecological risk assessment in a regulatory context. Environ Sci Technol. 38:463A-470A.

2. Posthuma L, Suter GW II, Traas TP. eds. 2002. Species-Sensitivity Distributions in Ecotoxicology. Lewis, Boca Raton, FL, USA.

3. Van Straalen NM, Denneman CAJ. 1989. Ecotoxicological evaluation of soil quality criteria. Ecotoxicol Environ Saf. 18:241-251.

4. Klepper O, Bakker J, Traas TP, Van de Meent D. 1998. Mapping the potentially affected fraction (PAF) of species as a basis for comparison of ecotoxicological risks between substances and regions. J Hazard Mater. 61:337-344.

5. Traas TP, Van de Meent D, Posthuma L, Hamers T, Kater BJ, De Zwart D, Aldenberg T. 2002. The potentially affected fraction as a measure of ecological risk. In Posthuma L, Suter GW II, Traas TP, eds, Species-Sensitivity Distributions in Ecotoxicology. Lewis, Boca Raton, FL, USA, pp 315-344.

6. Posthuma L, Traas TP, De Zwart D, Suter GW II. 2002. Conceptual and technical outlook on species-sensitivity distributions. In Posthuma L, Suter GW II, Traas TP, eds, Species-Sensitivity Distributions in Ecotoxicology. Lewis, Boca Raton, FL, USA, pp 475-510.

7. De Zwart D, Posthuma L. 2005. Complex mixture toxicity for single and multiple species: Proposed methodologies. Environ Toxicol Chem. 24:2665-2676.

8. Oreskes N, Shrader-Frechette K, Belitz K. 1994. Verification, validation, and confirmation of numerical models in the earth sciences. Science. 263:641-646.

9. Emans HJB, van de Plassche EJ, Canton JH, Okkerman PC, Sparenburg PM. 1993. Validation of some extrapolation methods used for effect assessment. Environ Toxicol Chem. 12:2139-2154.

10. Okkerman PC, van de Plassche EJ, Emans HJB, Canton JH. 1993. Validation of some extrapolation methods with toxicity data derived from multiple species experiments. Ecotoxicol Environ Saf. 25:341-359.

11. Posthuma L, Van Gestel CAM, Smit CE, Bakker DJ, Vonk JW. 1998. Validation of toxicity data and risk limits for soils. Final Report. 607505 004. National Institute for Public Health and the Environment, Bilthoven, The Netherlands.

12. Versteeg DJ, Belanger SE, Carr GJ. 1999. Understanding single-species and model ecosystem sensitivity: Data-based comparison. Environ Toxicol Chem. 18:1329-1346.

13. Posthuma L, Schouten AJ, Van Beelen P, Rutgers M. 2001. Forecasting effects of toxicants at the community level. Four case studies comparing observed community effects of zinc with forecasts from a generic ecotoxicological risk assessment method. In Rainbow PS, Hopkin SP, Crane M, eds, Forecasting the Environmental Fate and Effects of Chemicals. John Wiley, Chichester, UK, pp 151-175.

14. Giddings JM, Brock TCM, Heger W, Heimbach F, Maund SJ, Norman SM, Ratte HT, Schafers C, Streloke M. eds. 2002. Community-Level Aquatic Systems Studies-Interpretation Studies: CLASSIC. SETAC, Pensacola, FL, USA.

15. Smit CE, Schouten AJ, Van den Brink PJ, Van Esbroek MLP, Posthuma L. 2002. Effects of zinc contamination on a natural nematode community in outdoor soil mesocosms. Arch Environ Contam Toxicol. 42:205-216.

16. Van den Brink PJ, Brock TCM, Posthuma L. 2002. The value of the species-sensitivity distribution concept for predicting field effects: (Non-)confirmation of the concept using semifield experiments. In Posthuma L, Suter GW, II, Traas TP, eds, Species-Sensitivity Distributions in Ecotoxicology. Lewis, Boca Raton, FL, USA, pp 155-198.

17. De Zwart D, Dyer SD, Posthuma L, Hawkins CP. 2006. Use of predictive models to attribute potential effects of mixture toxicity and habitat alteration on the biological condition of fish assemblages. Ecol Appl (in press).

18. Dyer SD, White-Hull CD, Carr GJ, Smith EP, Wang X. 2000. Bottom-up and top-down approaches to assess multiple stressors over large geographic areas. Environ Toxicol Chem. 19:1066-1075.

19. Rankin ET. 1989. The Qualitative Habitat Evaluation Index (QHEI): Rationale, methods, and 
application. Ohio Environmental Protection Agency, Division of Water Quality Planning and Assessments, Columbus, OH, USA.

20. U.S. Environmental Protection Agency. 1992. Permit compliance system user documentation master index. PCS-MA93-1.01. Washington, DC.

21. U.S. Environmental Protection Agency. 1995. STORET/BIOS/ ODES/WQAS Tool Inventory. Washington, DC.

22. Wang X, White-Hull CE, Dyer SD, Yang Y. 2000. GIS-ROUT: A river model for watershed planning. Environment and Planning. B. Planning and Design. 27:231-246.

23. Little RJA, Rubin DB. 2002. Statistical Analysis with Missing Data. John Wiley, New York, NY, USA.

24. Dyer SD, Wang X. 2002. A comparison of stream biological responses to discharge from wastewater treatment plants in high and low population density areas. Environ Toxicol Chem. 21:1065-1075.

25. Stoddard JL, Larsen P, Hawkins CP, Johnson RK. 2005. Setting expectations for the ecological condition of running waters: The concept of reference condition. Ecol Appl (in press).

26. Ohio Environmental Protection Agency. 1996. Dissolved metals criteria. Ohio EPA Great Lakes Initiative Issue Paper, July 1996. Columbus, $\mathrm{OH}$.

27. U.S. Environmental Protection Agency. 1999. 1999 Update of ambient water quality criteria for ammonia. EPA-822-R-99-014. Office of Water, Washington, DC.

28. U.S. Environmental Protection Agency. 2002. ECOTOXicology Database System. Washington, DC.

29. van de Plassche EJ, de Bruijn JHM, Stephenson RR, Marschall SJ, Feijtel TCJ, Belanger SE. 1999. Predicted no-effect concentrations and risk characterization of four surfactants, linear alkyl benzene sulfonate, alcohol ethoxylates, alcohol ethoxylated sulfates, and soap. Environ Toxicol Chem. 18:2653-2663.

30. De Zwart D. 2002. Observed regularities in SSDs for aquatic species. In Posthuma L, Suter GW, II, Traas TP, eds, Species-Sensitivity Distributions in Ecotoxicology. Lewis, Boca Raton, FL, USA, pp $133-154$.

31. Shannon CE, Weaver W. 1949. The Mathematical Theory of Communication. University of Illinois, Urbana, IL, USA.

32. Moss D, Furse MT, Wright JF, Armitage PD. 1987. The prediction of the macroinvertebrate fauna of unpolluted running water sites in Great Britain using environmental data. Freshw Biol. 17:41-52.

33. Hawkins CP, Carlisle DM. 2001. Use of predictive models for assessing the biological integrity of wetlands and other aquatic habitats. In Rader R, Batzer DP, Wissinger SA, eds, Bioassessment and Management of North American Freshwater Wetlands. John Wiley, New York, NY, USA, pp 59-83.

34. McCullagh P, Nelder JA. 1989. Generalized Linear Models, 2nd ed. Chapman \& Hall, London, UK.

35. Trautman MB. 1981. The Fishes of Ohio, 2nd ed. The Ohio State University, Columbus, OH, USA.

36. Barbour MT, Gerritsen J, Snyder BD, Stribling JB. 1999. Rapid bioassessment protocols for use in streams and wadeable rivers: Periphyton, benthic macroinvertebrates, and fish, 2nd ed. EPA 841-B99-002. U.S. Environmental Protection Agency, Office of Water, Washington, DC.

37. Solomon KR, Takacs P. 2002. Probabilistic risk assessment using species-sensitivity distributions. In Posthuma L, Suter GW II, Traas TP, eds, Species-Sensitivity Distributions in Ecotoxicology. Lewis, Boca Raton, FL, USA, pp 285-313.

38. Mulder C, Cohen JE, Setälä H, Bloem J, Breure AM. 2005. Bacterial traits, organism mass, and numerical abundance in the detrital soil food web of Dutch agricultural grasslands. Ecol Lett. 8:80-90.

39. Giddings JM, Gonzales-Valero JF, Warren-Hicks WJ. 2003. Integrating concentration-response with species-sensitivity distributions. Abstracts, 24th Annual Meeting, SETAC North America, Austin, TX, USA, November 9-13, pp 297.

40. Klepper O, Traas TP, Schouten AJ, Korthals GW, De Zwart D. 1999. Estimating the effect on soil organisms of exceeding no-observed-effect concentrations (NOECs) of persistent toxicants. Ecotoxicology. 8:9-21. 